\title{
CLAUDIO ESTEVA, PROMOTOR DE LA ANTROPOLOGÍA EN MADRID. RECUERDOS DE UN TESTIGO
}

\author{
Fermín del Pino-DíAz \\ Consejo Superior de Investigaciones Científicas \\ fermindelpino@gmail.com
}

Como es bien conocido, el joven Claudio Esteva Fabregat (noviembre de 1918-septiembre de 2017) se exilió al final de la guerra civil española en México, donde formaba parte de un contingente numeroso de españoles jóvenes acogidos por aquel Gobierno, que los empleó laboralmente tras ayudarlos a completar su formación. Fue así que Claudio Esteva asistió, en primer lugar como alumno, a la Escuela Nacional de Antropología e Historia junto con otros exilados jóvenes (Ángel Palerm, Pedro Carrasco, Pedro Armillas, José Luis Lorenzo...) y, posteriormente, lo contrató el mismo centro para desarrollar en concreto el Área de Cultura y Personalidad, como confiesa el propio autor en sus entrevistas autobiográficas.

Han sido varias las personas que han relatado todo su recorrido formativo. Estas han tomado la información de labios del propio autor, relatos que se pueden consultar en línea o en homenajes diversos que se le han dedicado merecidamente ${ }^{1}$. Ahora nos falta, tal vez, reunir una ver-

1 Recomiendo leer el esfuerzo de contexto histórico-científico de su compañero en la Universidad de Barcelona, el geógrafo e historiador de la ciencia Horacio Capel (Capel, 2009). 
sión ajena, a partir de personas que, o bien no tuvieron un contacto directo con él, o bien no pertenecieron a su entorno personal. A mi juicio, este segundo testimonio ofrece un valor añadido de objetividad o, al menos, de eco institucional y colectivo que muestra la profunda huella que dejó su influencia. Ahora que la cruel suerte de los ancianos se lo ha llevado consigo (a punto de cumplir cien años, como el propio Lévi-Strauss) es hora de mirar atrás y analizar la influencia institucional de don Claudio, sobre todo en el ámbito de la disciplina antropológica. Tanto su origen como destino final en Cataluña han motivado que la mayor parte del reconocimiento de su labor se haya concentrado en manos de los antropólogos vinculados de alguna manera a la Universidad de Barcelona, pero es justo decir que su influencia también se percibe en el americanismo hispano y en la antropología de Madrid, aunque allí residiera menos tiempo, entre su salida mexicana en 1956 hasta su traslado a Barcelona en 1969.

Así las cosas, sobre lo que cabe aún explayarse es respecto a su contribución particular al desarrollo institucional antropológico, especialmente en Madrid. En la capital española retomó los estudios universitarios recién llegado de México - obtuvo la licenciatura de Filosofía y Letras en tan solo un año-y se incorporó casi inmediatamente al claustro de profesores en el Departamento de Historia de América. Yo lo conocí a los diez años de su llegada, en 1966, como director del Museo Nacional de Etnología (MNE) y secretario general del Centro Iberoamericano de Antropología (CIDA), mientras yo cursaba el segundo curso en este centro (1966-1967), y debo decir que no hubiera podido integrarme en el gremio antropológico sin la mediación de don Claudio. Efectivamente, fue él quien me aceptó como alumno en el CIDA tras una entrevista personal facilitada por mi director de tesis doctoral, Juan Pérez de Tudela, que a la sazón era uno de los profesores de aquel centro. Don Claudio también me invitó al año siguiente, en mayo de 1968, a dictar un seminario de dos semanas sobre indigenismo y antropología aplicada en el propio CIDA y quien me propuso para integrarme en el año académico 1968-1969 en el claustro de profesores de la Facultad de Filosofía y Letras como encargado del curso de Antropología y Etnología de América. De nuevo, don Claudio me planteó en 1976 la coordinación del Simposio de Historia de 
la Antropología en el Primer Congreso Nacional de Antropología, que él coordinaba desde la Universidad de Barcelona. Justamente allí tomé más consciencia de que don Claudio formaba parte del gremio de los antropólogos exilados, a algunos de los cuales invitó a dar su testimonio con ocasión del mencionado evento. Muchos de ellos formaban parte del profesorado en el CIDA, como Juan Comas y Santiago Genovés, y, en general los allí invitados eran - a mi modo de ver- más próximos a su enfoque con respecto a la teoría antropológica cultural. Genovés volvía a coincidir con Claudio, así como Ángel Palerm, que, desde unos años atrás, venía con frecuencia a Madrid y Barcelona y, sobre todo, Pedro Carrasco, que continuaba su labor en universidades norteamericanas y seguía ejerciendo una enorme influencia sobre la antropología cultural mexicana. Si mal no recuerdo, creo que fueron justamente Comas, Genovés, Pedro Armillas y Pedro Carrasco quienes contribuyeron en 1964 a crear el CIDA, como veremos enseguida

\section{Labor inicial en España en favor de la antropología americana}

Por las razones que fuera, don Claudio quiso regresar a España muy pronto, tras menos de veinte años de exilio. No acabo de entender los motivos expuestos por el autor en su autobiografía (continuación de la lucha política socialista, que lo había llevado al exilio) y pienso que tuvo que ver más bien con la nueva política mexicana hacia los exilados (nacionalización obligatoria, competencia profesional con los nacionales) lo que llevó a muchos a buscar un nuevo lugar fuera de México, como les ocurrió a Palerm, Armillas, Carrasco, entre otros antropólogos. De hecho, no le conocí, tras su vuelta, actividad política alguna a don Claudio, lo que, al mismo tiempo, me parece difícilmente conciliable con el ascenso institucional que protagonizó desde sus primeros años en España, entre 1956 y 1964. Alumno de la Facultad de Filosofía y Letras durante uno o dos cursos en la especialidad de Historia de América, pronto comenzó a dictar clases en ese departamento, dirigido por don Manuel Ballesteros, y a colaborar en las investigaciones americanistas del Consejo Superior 
de Investigaciones Científicas (CSIC), más concretamente en el Instituto Fernández de Oviedo, dirigido por don Ciriaco Pérez Bustamante.

Con todo, lo evidente es que su vocación de antropólogo mexicanista, gestada en su larga estancia en el país americano, se volvió a confirmar desde su llegada a España. Su actividad fundamental se desarrolló alrededor de dos temas principales del indigenismo mexicano: las culturas mesoamericanas y la evolución del mestizaje indo-hispano. También le interesaba el tema de la antropología aplicada, muy desarrollada en México dentro del indigenismo oficial, e incluso lo contrataron en España para estudiar cuestiones socioeconómicas de la sociedad agraria, en particular, en zonas de colonización meridional, así como de la España colonial, en Guinea Ecuatorial. Por otro lado, don Claudio - como americanista y miembro activo de una disciplina que comenzaba a dar sus primeros pasos de manera autónoma— no dejó nunca de interesarse por cuestiones meramente teóricas, como la historia de la antropología en España y su identidad disciplinar frente a la sociología y otras ciencias próximas, como la etnología, prehistoria, arqueología, demografía, entre otras. A juzgar por sus publicaciones, muchas de las cuales eran reseñas de trabajos antropológicos coetáneos y juzgadas frecuentemente como «metodológicas», su obra evidenciaba un claro énfasis «disciplinar» antropológico (Esteva, 1957; Esteva, 1959; Esteva, 1963; Esteva, 1964; Esteva, 1969a; y Esteva, 1969b).

Aunque no pude asistir al evento personalmente en 1964, quiero poner el acento en una actuación de don Claudio que, a mi entender, marcó su destino inmediato en la vida disciplinar española y que evidenció su orientación americanista. Me refiero al XXXVI Congreso Internacional de Americanistas, celebrado en varios lugares, entre otras ciudades españolas, Barcelona y Madrid, aunque destacaron las actividades que tuvieron lugar en Sevilla. Este tipo de congresos internacionales había surgido en el siglo xix de la mano de autoridades europeas, especialmente francesas, dedicadas al estudio de las antigüedades americanas (lenguas, arqueología, prehistoria, demografía, historia de los descubrimientos y sus fuentes...). El primero de estos eventos se había organizado en Nancy a iniciativa del americanista Edward Hamy y, desde entonces, en aquel 
siglo solo se celebró en España dos veces: el IV en Madrid (1881) y el IX en Huelva (1892). En el siglo xx tuvo lugar otro magnífico congreso, el XXVI, celebrado en Sevilla, en el que se mostró un claro interés por las obras del P. Las Casas ${ }^{2}$. En aquella época, hubo varios nombres destacados entre los americanistas españoles. Del siglo xix, cabe señalar a Cayetano Fabié y Marcos Jiménez de la Espada; por su parte, del siglo siguiente no se puede olvidar a Antonio Ballesteros, quien trabajó sobre asuntos colombinos particularmente, y Rafael Altamira, jurista discípulo de Joaquín Costa, que se interesó por el estudio de las costumbres locales y la metodología histórica. Uno de los más jóvenes españoles destacados en el congreso de Sevilla fue el historiador Ramón Iglesia, que pronto se trasladaría a México como exilado, donde desplegaría en pocos años una labor docente notable; para ello, fundó en el Colegio de México y en universidades norteamericanas una escuela de alumnos interesados en el estudio de las fuentes españolas de América (Bernal, Gómara, Mendieta...). En el mencionado congreso de la capital hispalense estuvieron presentes otros miembros de la Universidad y del Centro de Estudios Históricos (una de las instituciones de la Junta para Ampliación de Estudios) interesados en el Nuevo Mundo; entre ellos, algunos becados extranjeros en España, como Silvio Zavala o Ángel Rosenblatt. Destacó asimismo la presencia del joven Raúl Porras, que presentó tres ponencias y logró convencer a los congresistas para que la siguiente sesión americanista se celebrase en Lima, que tendría lugar en 1937. Desde comienzos del nuevo siglo, los americanistas lograron llevar al Nuevo Mundo la celebración de tan reputado encuentro cada cuatro años ${ }^{3}$.

Así las cosas, en los años sesenta, don Claudio asistiría a tres congresos americanistas: Viena, en 1960, México, en 1962 y Sevilla, en 1964. Fue testigo de la escasa presencia institucional de la antropología española en el extranjero y del papel innovador asociado a nombres de españoles

2 Un detalle del tono republicano de nuestro americanismo oficial de entonces es que, cuando las autoridades norteamericanas trajeron a Trujillo en 1929 y a Lima en 1935 la conocida estatua de Pizarro, la embajada española en Lima propuso situar a su lado un busto del Inca Garcilaso (véase mi artículo, Pino 2016).

3 Los otros dos congresos internacionales de americanistas celebrados en España fueron el LII, en Sevilla (2006), y el LVI, que se celebró en Salamanca en 2018. España es uno de los países donde más congresos de esta serie se han celebrado. 
exilados en México. La presencia de españoles que investigaban en España en esos congresos alcanzó la decena justamente a partir del congreso de Viena: «... de ese congreso [...] se aprecia a partir de 1960 un sensible aumento respecto a la década anterior que viene a representar, en promedio, el doble que en el primer período de tiempo considerado» (Alcina, 1972: 32). Es verdad que por esos años asistiría don Claudio igualmente a otros congresos antropológicos, tanto internacionales como nacionales ${ }^{4}$. Pero realmente fue el americanismo el ámbito en que se desarrolló principalmente la antropología española y, por eso, el congreso de 1964 tuvo lugar en las tres ciudades americanistas por excelencia: Sevilla, Madrid y Barcelona. Allí se habían fundado los seminarios de estudios indigenistas en las décadas de 1950 y 1960, que incluyeron posteriormente cátedras de antropología, arqueología y prehistoria.

Don Claudio tuvo una presencia activa en el XXXVI Congreso Internacional de Americanistas. Gracias a él se dedicó un monográfico de la Revista de Indias, el número 24, al mestizaje hispanoamericano. La publicación constituía el órgano del Instituto Fernández de Oviedo, que a la sazón había sido uno de los principales coordinadores institucionales del congreso americanista. Lo importante, según creo, fue la coincidencia masiva de americanistas españoles en el encuentro, especialmente de antropólogos de procedencia exilada (Comas, Genovés, Díaz Hungría, Armillas, Carrasco, Esteva...). Aunque otros asistentes no eran españoles (Matos Mar, John V. Murra, María Rostworowski), muchos de ellos impulsaron la creación de institutos antropológicos nacionales, como el prestigioso Instituto de Estudios Peruanos, fundado al tiempo que el CIDA. Coetánea también fue la creación de la sección antropológica de la Universidad Iberoamericana de México, gracias a la labor de Ángel Palerm, otro exilado español que, justamente, había regresado poco tiempo antes de la Oficina de Asuntos Sociales de la Organización de los Estados Americanos, donde había colaborado activamente en la traducción

\footnotetext{
4 X Reunión Internacional del Centro Europeo de Documentación e Información, Madrid, 1961; Congrès International de Scienciences Anthropologiques et Ethnologiques, París, 1963; Primer Congreso de Etnología y Tradiciones Populares, Zaragoza, 1968.
} 
de manuales antropológicos, como el famoso texto Notes and Queries del Royal Anthropological Institute ${ }^{5}$.

A pesar de lo apuntado hasta el momento, no se fundó en España un centro específico de antropología, la Escuela de Estudios Antropológicos (EEA), hasta 1965. Formaba parte del CIDA y estuvo adscrita al Instituto de Cultura Hispánica (ICH) por decisión de sus autoridades y a petición de los asistentes al XXXVI Congreso Internacional de Americanistas, el que se había celebrado un año antes de la creación de la EEA. A raíz de una decisión del congreso americanista, se estableció que don Ciriaco Pérez Bustamante fuera el presidente del CIDA y Claudio Esteva su secretario general (en concreto, ambos formaban parte de su patronato, compuesto por representantes de varias instituciones americanistas), quienes se habían mostrado como miembros muy activos en el congreso americanista, adonde habían asistido autoridades del ICH como su presidente, Gregorio Marañón, y su jefe de comunicación, José M. ${ }^{a}$ Álvarez Romero.

La EEA se ubicó precisamente en el MNE y en 1965 nombraron a su nuevo director, Claudio Esteva, tras la reciente renuncia de Julio Caro Baroja, desesperado por la falta de apoyo institucional. En realidad, don Julio no había demostrado nunca mucha afición por los estudios americanistas (véase Pino, 1996b; recogido en Müllauer-Seichter, 2016: 109-132). El mencionado nombramiento, el de secretario general del CIDA, coincidió con la constitución de la EEA. Ante esta situación, no cabe disimular la conexión entre ambos cargos ni la deriva del congreso americanista del año anterior, donde había quedado patente la debilidad institucional española, hecho que había impulsado la nueva creación nacional. Ya había ocurrido previamente una fundación similar a comienzos del siglo xx, cuando poco después de la celebración de un congreso internacional de antropología se creó la Sociedad Española de Antropología.

La EEA — de corta existencia, entre 1965 y 1968 - guarda una estrecha relación con don Claudio, su director único durante los tres años, hasta su nombramiento como profesor agregado de Etnología en la Universi-

5 Hay referencias en el Archivo de la Agencia Española de Cooperación y Desarrollo (nuevo nombre del antiguo ICH) sobre la búsqueda de este material antropológico traducido, procedente de Palerm, por parte del Centro Iberoamericano. Está por estudiar, en base a estos archivos conservados del CIDA, el proceso de creación de la anexa EEA. 
dad de Barcelona en marzo de 1968. Puedo testimoniar —como simple miembro- el funcionamiento e influencia de la escuela en la antropología española. Creo que mi testimonio puede tener algún interés, a la espera de un estudio más minucioso (por mi parte o la de alguno de los otros miembros que formamos parte de ella). Quisiera destacar algunos nombres de las tres promociones de alumnos. De la primera, de 1965, recuerdo a Pilar Romero de Tejada, con la que guardo cierto parentesco y quien sería conservadora y directora del MNE, así como a Manuel Gutiérrez, posteriormente, catedrático de Antropología Americana. De la segunda, a la que pertenezco, mis recuerdos son, lógicamente, más minuciosos: Isidoro Moreno y Pilar Sanchís (de Sevilla), Miguel Rivera y Rogelio Rubio (de Madrid), Josefina Roma (de Barcelona) y algún becario americano como Sara Roitman (de Argentina). De la tercera promoción recuerdo especialmente a Enrique Luque, Salvador Rodríguez, Cuadrat Ciurana y Joaquín Puig. Creo que casi todos ellos han proseguido una carrera antropológica, aunque no siempre como profesión principal. Media docena de ellos han obtenido una cátedra de Antropología y puede afirmarse que los departamentos de Madrid, Barcelona y Sevilla se nutrieron en sus inicios de esta primera generación formada por Claudio Esteva en la década de 1960.

\section{La Escuela de Estudios Antropológicos}

Puede afirmarse, sin miedo alguno a errar, que en España no se enseñó formalmente antropología social o cultural hasta la instauración en 1965 de la escuela sita en Atocha, donde se ubicaba el MNE desde su creación en 1875 y el lugar en que se estableció la sede de la escuela dirigida por Claudio.

En realidad, algo de esas disciplinas ya habían enseñado quienes fueron miembros del museo, como el doctor Velasco, Manuel Antón, Barras de Aragón, Luis de Hoyos o su colega Telesforo de Aranzadi. En todo caso, se trataba de enseñanzas que complementaban la comparación anatómica de las razas humanas; la disciplina de formación de todos ellos era la zoología o la medicina. Lo más cercano a la etnología que hasta entonces se practicó en la Península fue posiblemente el derecho consuetudina- 
rio, coordinado por miembros ilustres de la escuela del aragonés Joaquín Costa, como Rafael Altamira, Manuel Pedregal, Juan Serrano, Dorado Montero o Miguel de Unamuno, entre otros, o el folklore de la escuela del sevillano Machado y Álvarez, especialmente impulsada por su paisano Rodríguez Marín. Todos ellos, según puedo hacer constar, procedían del derecho (Pino 1996b).

Por otro lado, lo más parecido a la antropología social que tenía lugar en tiempos de la escuela de Atocha eran los cursos de sociología de la Facultad de Derecho y de Ciencias Políticas, de donde procedíamos varios de los alumnos de la EEA, como M. Gutiérrez, Rogelio Rubio, F. del Pino, Joaquín Puig y Cuadrat Ciurana. Allí, se trataban cuestiones de antropología social, si bien marginalmente (apenas emergían los nombres ilustres de Durkheim, Malinowski y Lévi-Strauss). La progresiva presencia en España de la editorial mexicana Fondo de Cultura Económica difundía en esas fechas algunos de los manuales norteamericanos, como el de Clyde Kluckhohn (1961), que era lo único que yo había leído por mi cuenta. Por primera vez se anunciaron en la prensa madrileña y a través de folletos los cursos de antropología que iban a dictarse en el MNE, que incluían Antropología Social y Cultural, Arqueología, Etnohistoria, Prehistoria, Antropología Física, Demografía, Lingüística y una serie de cursos sobre historia y geografía de España e Iberoamérica. En la información disponible se anunciaba que el número máximo de alumnos sería de entre 20 y 25 , la mitad de ellos españoles y la otra mitad americanos, que la mayoría estarían becados, que el horario sería vespertino y que tendría una duración total de nueve meses, con un trabajo de campo obligatorio y un informe final. Se exigía tener aprobada una licenciatura previa (Filosofía y Letras, Sociología, Ciencias Políticas, Derecho, etc.) y aprobar un examen de acceso para determinar el conocimiento previo del candidato respecto al área de estudio y su motivación para matricularse.

Las primeras promociones se nutrieron, como es lógico, de alumnos que ya conocían los profesores invitados; incluso algunas facultades o seminarios contaron con un cupo de candidatos posibles (me consta que así era, al menos, para los estudiantes de Sevilla). Yo me inscribí en esta escuela a raíz de que mi director de tesis, Pérez de Tudela, (con quien me 
propuse conocer mejor la figura del P. Las Casas), me informara y, junto con una docena de candidatos aproximadamente (licenciados de Sevilla, Barcelona, Madrid y alguno de Perú, Guinea y Estados Unidos), superé una entrevista con don Claudio.

La beca de 6000 pesetas mensuales era más que suficiente para mis expectativas como joven licenciado que deseaba ampliar sus estudios. Otra beca doctoral que obtuve al término de la escuela ascendía a un poco más, 10000 pesetas, pero el sueldo de un encargado de curso universitario, puesto que empecé a desempeñar en 1968, no superaba las 5000 pesetas. Por su parte, el sueldo de nuestros profesores de la escuela ascendía a 1000 pesetas por hora de clase. Se trataba de un plan de estudios bien dotado, a imitación del republicano Centro de Estudios Históricos o del posterior modelo mexicano del Colegio de México. Estoy seguro de que el modelo mexicano, gestado en contacto con los profesores españoles alojados en la longeva Casa de España (luego Colegio de México) ejerció alguna influencia. Entre los profesores que vinieron varios años a impartir cursos a la escuela se encontraban varios mexicanos, como Jiménez Moreno, León Portilla, Santiago Genovés, Juan Comas, etc., algunos de los cuales eran exilados españoles. Yo creo que era evidente la influencia de algunos de ellos a la hora de gestar la fundación de la escuela en la esfera del congreso americanista de 1964, donde se encontraron y reforzaron los lazos de amistad previos surgidos durante su exilio mexicano. Varios de ellos eran colegas, estudiantes o profesores, de la Escuela Nacional de Antropología e Historia de México.

El temario sobre antropología propuesto en la escuela era novedoso por su espíritu interdisciplinar y en todas las clases impartidas se traían a colación ejemplos de sociedades diversas y diferentes épocas con el objetivo de comparar, siempre presente en todas las materias. Era diversa la procedencia, disciplinar y geográfica, de los profesores y de los alumnos. Cada profesor dictaba su curso de dos horas diarias durante una semana y reclamaba generalmente la participación de los asistentes, a veces con un ejercicio escrito. Con frecuencia se suscitaban debates entre los alumnos y los profesores, y siempre se procuraba consultar una referencia bibliográfica en la biblioteca del museo, en proceso de renovación a instancias 
de don Claudio. Como es habitual, los ejercicios escritos recibían del profesor un informe minucioso de su calidad y expectativa profesional esperada (aún se conservan esos exámenes, manuscritos o mecanografiados). Algunos temas que recibieron especial atención eran los relacionados con cultura y personalidad (promovidos por Esteva), con Lévi-Strauss y el estructuralismo (abordados por Lisón), así como con casos de antropología aplicada (especialmente, del campo del indigenismo mexicano, tratados por León Portilla y Jiménez Moreno) e, incluso, se estudiaron las novelas del peruano Castañeda relacionadas con el peyote mexicano y otras drogas tradicionales, como Las aventuras de don Juan. Algunos profesores exilados como Santiago Genovés nos alentaban a debatir sobre la antigüedad del hombre americano o las bases biológicas de la violencia humana. Otros, como Manuel Alvar, suscitaban debates de lingüística como el método de palabras-cosas, los mapas lingüísticos o el estructuralismo.

Algunas de las clases tenían una parte práctica, como en el caso de Antropología Física, donde se nos enseñaba a medir un cráneo o tomar una muestra de grupo sanguíneo; asimismo recibíamos clases prácticas para clasificar objetos del museo anexo a la escuela. El curso de tres trimestres, a razón de cinco horas diarias de clase, concluía con un trabajo de campo de un mes. Cada alumno se desplazaba a una comunidad rural: varios estudiantes de primer cursos fueron al pueblo de Buitrago; otros alumnos del mismo año visitaron individualmente una comunidad distinta pero cercana, como Villasequilla, Corpa, etc.; la segunda promoción, de la que formaba parte, fuimos a Bielsa, en los Pirineos aragoneses, y a la docena que formábamos el grupo nos ubicaron en ocho pueblos de la comarca (a mí me tocó Gistaín, junto a la sevillana Pilar Sanchiz). En todos los casos, los alumnos debían entregar un trabajo de fin de curso de un centenar de páginas, donde debía reflejarse su experiencia, método y datos recogidos. Ignoro el trabajo de campo de la tercera promoción de la escuela, que se continuó en el entorno de Bielsa y que seguiría siendo objeto de atención de don Claudio desde Barcelona, pero sé que la comarca oscense ha sido objeto de visitas asiduas de los alumnos catalanes de don Claudio y ha constituido un área de especial interés etnográfico posteriormente. 


\section{Cierre de la escuela y difusión nacional posterior}

Al mismo tiempo que don Claudio focalizaba su atención en el museo y la escuela, su propia carrera académica no se interrumpía y, así, logró en febrero de 1968 el puesto de profesor agregado de Etnología en la Universidad de Barcelona, que vería convertido poco después en cátedra de Antropología Cultural. La correspondencia epistolar que envió Claudio desde la escuela muestra la preocupación por esa tarea desde el otoño de 1967. En una carta enviada al geógrafo Benito Arranz explica que las funciones de la escuela las continuaría dirigiendo el nuevo Departamento de Antropología de América, diferente del de Historia de América —aunque luego se acabaría llamando simplemente de Historia de América II-, constituido entonces en la Facultad de Filosofía y Letras de la Complutense, como sucesor del antiguo Seminario de Indigenismo, patrocinado en la década de 1950 por M. Ballesteros. Para don Claudio, el programa de esta subsección de antropología y etnología de América era «una copia del de la escuela». Efectivamente, en el nuevo entorno universitario encontramos a varios de los profesores de la escuela, como Ballesteros, Alcina, Carmelo Lisón, M. Gutiérrez, Rogelio Rubio y yo mismo, y es verdad que luego se alentó que se realizaran trabajos de investigación, tanto en América como en comunidades españolas. Desgraciadamente, cuando la escuela estaba adquiriendo el reconocimiento como centro de referencia internacional, los cursos se suspendieron súbitamente al comienzo del curso 1968-1969 y los profesores, contratados y alumnos -incluso quienes ya habían recibido la beca para ese mismo año- recibieron una carta del 27 de setiembre de 1968 en que se comunicaba lo siguiente: «Debido a una reorganización del CIDA, han sido suspendidos los cursos del período académico 1968-1969 de la Escuela de Estudios Antropológicos». Aunque desde un punto de vista se trataba de la apertura a un desarrollo autónomo de la Universidad española, lo cierto es que desaparecía una institución centralizada y de referencia internacional. Había consistido hasta entonces en un lugar de visita asegurada de profesores venidos de muy diversos lugares, de debate intenso entre promociones de licenciados de disciplinas diversas, de trabajo de campo conjunto o de preocupación compartida por las novedades teóricas y los posicionamientos polémicos. 
Y, con ella, desapareció el privilegio de las becas que recibían nacionales y extranjeros, quienes conformaban, cada promoción, un grupo de cerca de una docena de licenciados de diversa procedencia. En resumen, toda esta riqueza se convirtió, simplemente, en una licenciatura universitaria.

Es verdad que en aquel momento comenzaron a impartirse asignaturas de Antropología Social y Cultural en los departamentos de Filosofía y Letras de otras ciudades, especialmente en Barcelona y Sevilla, así como en la nueva Facultad de Ciencias Políticas y Sociología, donde comenzó a abrirse un espacio a la antropología social de la mano de Carmelo Lisón. Gracias a nuevas subvenciones de fundaciones nacionales (especialmente de la Fundación Juan March) surgieron oportunidades para que algunos alumnos interesados estudiaran en el extranjero. Los departamentos universitarios comenzaron a enviar y a recibir antropólogos formados fuera. El dinamismo antropológico centralizado, aunque diverso y cambiante, generado por la escuela de Atocha, pasó a ser sustituido por una fragmentación universitaria e, incluso, - como era de esperar- se hizo patente un enfrentamiento entre quienes sostenían versiones diversas de la propia disciplina, patrocinadas por diferentes catedráticos. Empezó a llegar a España el debate suscitado entre la antropología inglesa y la norteamericana sobre la supuesta diferencia de calidad entre la antropología social y la cultural, al que don Claudio era sensible, o, asimismo, entre los partidarios de una antropología marxista cercana a la gran teoría social y los críticos posmodernos, partidarios del estudio de casos o de matices intersubjetivos e históricos. Los sociólogos empezaron a acusar a la antropología de colaboradora del colonialismo e, incluso, algunos jóvenes profesionales - orgullosos de la autonomía política de su región- comenzaron a acusar a los antropólogos de imperialismo y desviamiento ideológico. Se trataba de un argumento particularmente injusto hacia quienes habían contribuido a la formación de un corpus etnográfico nacional antes inexistente y hacia quienes ofrecían oportunidades excepcionales de formación en una disciplina antes no practicada. Pero resultó ser un argumento exitoso, especialmente útil a profesionales jóvenes que no querían competencia disciplinar ajena en su propio territorio. 
Precisamente la nueva situación política de las autonomías que estableció la nueva Constitución de 1978 favoreció que ciertos departamentos antropológicos recibieran apoyo y se aumentó el número de cátedras de Antropología Social y Cultural. Ello vino precedido de una multiplicación de asociaciones regionales de antropología, que tomaron desde $1981 \mathrm{el}$ relevo en el control de los congresos nacionales (Madrid, Granada, Zaragoza...) y dejaron a los maestros de la generación anterior, hasta cierto punto, desconectados del futuro profesional. En el caso de don Claudio, este salto generacional de la cadena de mando le llegó (como a otros como José Alcina, Alfredo Jiménez, Gustavo Bueno, Juan Pérez de Tudela, Carmelo Lisón...) en un momento cercano a su jubilación y, creo, le generó una especial frustración personal. En este sentido, no debe ser del todo independiente que buscara un nuevo horizonte territorial para orientar su jubilación fuera de España, como finalmente hizo, precisamente en México, donde había tenido lugar su iniciación antropológica.

Seguramente, debió sentir como una compensación personal que las instituciones mexicanas apreciaran el esfuerzo personal hecho en España, sin renunciar a su formación antropológica mexicana. De México regresó a Barcelona cuando las fuerzas le fallaron definitivamente, a edad muy avanzada. También la sociedad antropológica norteamericana lo había consagrado al concederle la medalla Malinowski en nombre de la Sociedad de Antropología Aplicada, un campo en que don Claudio había insistido siempre, así como en otros más novedosos, como la antropología industrial.

Si cabe la validez de una apreciación personal sobre don Claudio, mi iniciador disciplinar, considero que más que un especialista en un campo disciplinar determinado (aunque es evidente que Claudio tenía sus preferencias: el mestizaje, el nacionalismo, la subjetividad ante lo étnico), en lo que destacó a lo largo de su vida fue en su capacidad de concebir un futuro práctico para la antropología, en sus posibilidades de institucionalización. De hecho, sin él y su capacidad de mediar entre las autoridades académicas y políticas, de convencer de multitud de maneras (casi todas seductoras y bien argumentadas) sobre las posibilidades futuras de una disciplina a los que estaban en posición de decidir los presupuestos, no 
habría existido la EEA ni un departamento de Antropología Cultural en Barcelona (el primero que tuvo su revista, su congreso, sus proyectos de investigación, su cuerpo de profesores), que sirvió a modo de paradigma para el resto de las universidades.

Si Esteva Fabregat no hubiera ido de la Universidad de Madrid a la de Barcelona, es posible que el panorama nacional disciplinar no habría adoptado el perfil autonomista que tiene en la actualidad. Tal vez habríamos iniciado debates disciplinares internos para integrar las diversas academias regionales, lo que nos habría permitido ofrecer una versión más unificada y característica de la disciplina, como Reino Unido, Francia y Estados Unidos mostraron a través de sus maestros sucesivos, autores de sendas escuelas de organizaciones sociales propias de la disciplina (Malinowski, Lévi-Strauss o Franz Boas). Piénsese en lo que significó para el reconocimiento de una identidad intelectual nacional el surgimiento en Madrid de la escuela filosófica de Ortega o en Barcelona la arqueológica de Bosch Gimpera o la jurídica de Giner de los Ríos, difundida por toda España. Esto no quiere decir que esas otras escuelas antropológicas nacionales no hayan mantenido disputas y dinámicas internas de fragmentación, pero la conformación de una posición magistral inicial permitió gestar un relato propio de la disciplina, aunque solo fuera en calidad de rebelión discipular, que a nosotros nos ha faltado. 


\section{Bibliografía citada}

Alcina J. 1972. "La antropología americanista en España: 1950 -1970», Revista Española de Antropología Americana VII(1): 17-58. Madrid: Universidad Complutense.

CAPEL, H. 2009. «La antropología española y el magisterio de Claudio Esteva Fabregat. Estrategias institucionales y desarrollo intelectual en las disciplinas científicas», Scripta Nova. Revista Electrónica de Geografía y Ciencias Sociales XIII(287). Barcelona: Universidad de Barcelona. Recuperado de <http://www.ub.es/geocrit/sn/sn-287.htm>.

Esteva, C. 1957. «Sobre la teoría y los métodos de la antropología social», Revista Internacional de Sociología 59: 411-436. Madrid: CSIC.

Esteva, C. 1959. «Sobre el método de la arqueología», Revista de Indias 75: 89-106. Madrid: CSIC.

Esteva, C. 1963. «El método funcionalista en el estudio de la aculturación», VIe Congrès International des Sciences Anthropologiques et Ethnologiques II. París: Musée de l'Homme, 583-587.

Esteva, C. 1964. «Aculturación y mestizaje en Iberoamérica. Algunos problemas metodológicos». Revista de Indias XXIV(97-98): 445-472. Madrid: CISC.

Esteva, C. 1969a. «Problemas metodológicos de la antropología española», Primer Congreso de Etnología y Tradiciones Populares. Zaragoza. Institución Fernando el Católico, 41-52.

Esteva, C. 1969b. «Sobre el método y los problemas de la antropología estructural». Convivium 30:3-54. Barcelona: Universidad de Barcelona.

Müllauer-Seichter, W. 2016. Claves en los inicios de la antropología social y cultural española. Temas y autores. Madrid: Editorial Universitaria Ramón Areces.

Pino, F. del 2016. «La erección en España de tres estatuas de héroes, obra norteamericana», La Albolafia: Revista de Humanidadesy Cultura 7: 217-230. Pino, F. del 1996a. «¿Abogado o antropólogo? La obra etnológica de Joaquín Costa y su pluralidad disciplinar y contextual», VII Congreso de Antropología Social. Zaragoza: Instituto Aragonés de Antropología, 23-53.

PINo F. del 1996b. «Clasicismo y génesis disciplinar antropológica: el caso de Julio Caro Baroja», Revista de Dialectología y Tradiciones Populares LI(1): 303-330. Madrid: CSIC. 